\title{
Impairment of podocyte function by diphtheria toxin-a new reversible proteinuria model in mice
}

\author{
Andreas Goldwich ${ }^{1}$, Alexander Steinkasserer ${ }^{1}$, André Gessner ${ }^{2, *}$ and Kerstin Amann ${ }^{3, *}$
}

Diphtheria toxin (DTx) receptor (DTR)-mediated conditional cell ablation in transgenic mice is a powerful tool to analyze cell function in vivo. Transgenic mice with cell-specific expression of the human DTR have been developed that allow conditional depletion of these cells in vivo through administration of the toxin. We have performed a careful analysis of mice after DTx injection and found an unexpected side effect. Treatment of wild-type C57BL/6 mice with DTx leads to a marked transient and completely reversible proteinuria, as a consequence of podocyte dysfunction that is morphologically characterized by foot process fusion and detachment from the glomerular basal membrane. In vitro analysis displayed that DTx-treated podocytes show diminished attachment to basal membrane proteins. Five to 9 days after DTx application the mice recover completely. Glomerular proteinuria is a hallmark of glomerular disease due to dysfunction of the filtration barrier. Rodents have been extensively used experimentally to better define the mechanisms of disease induction and progression. However, nongenetic mouse models of proteinuric glomerular damage are limited and display various shortcomings. We suggest DTx-induced transient kidney dysfunction as a new reversible model of experimental podocyte injury, which could be used as an additional approach to complement studies in human.

Laboratory Investigation (2012) 92, 1674-1685; doi:10.1038/labinvest.2012.133; published online 24 September 2012

KEYWORDS: diphtheria toxin; mouse model; podocytes; proteinuria; slit membrane

Diphtheria toxin (DTx) is an exotoxin secreted by Corynebacterium diphtheriae, the pathogenic bacterium that causes diphtheria. The toxin gene is encoded by a bacteriophage and consists of homodimers linked by disulfide bridges of a polypeptide chain of 535 amino acids (about $62 \mathrm{kDa}$ ) and two subunits. The entry of the toxin, or at least the A-fragment, into the cytoplasm is required for the cytotoxic action. ${ }^{1}$ DTx causes inhibition of protein synthesis by inactivation of the eukaryotic elongation factor-2 (eEF2). As $\mathrm{NAD}^{+}$diphthamide ADP-ribosyltransferase the toxin catalyzes the ADP-ribosylation of eukaryotic eEF2. The sensitivity of cell to DTx varies among species. In sensitive species including man, guinea pigs, rabbits and many birds $50-100 \mathrm{ng} / \mathrm{kg}$ of DTx is lethal whereas mice and rats are far more, albeit not completely resistant. ${ }^{1}$ The difference is primary determined by the DTxspecific receptors on the cell surface. The DTx receptor (DTR) originally identified from Vero cells ${ }^{2}$ has been shown to be identical with the heparin-binding EGF-like growth factor. ${ }^{3}$

Recently, a novel DTx-based system was developed, which allows the short-term ablation of specific cells in vivo, called toxin receptor-mediated cell knockout. A single administration of DTx to transgenic mice that express the primate DT receptor under control of the target cell-specific promoter was found to result in depletion of these cells. ${ }^{4}$

The main function of the glomerulus is to filter blood plasma into primary urine for the subsequent handling by the renal tubular system. The glomerular filter resides in the glomerular capillary wall, which is composed of fenestrated endothelial cells, podocytes connected by filtrated slits and the intervening glomerular basement membrane (GBM). By this size and charge selective glomerular filter, large plasma proteins such as albumin are retained in the plasma. Thus, the presence of large plasma proteins in the urine reflects a damage at the glomerular filtration barrier (ie, glomerular proteinuria). Of note, mutations in several genes relevant to podocyte function are known to cause proteinuria and the nephrotic syndrome, ie, WT-1, podocin, nephrin, etc. ${ }^{5}$

Most animal models of nongenetic proteinuric glomerular disease have so far been induced in the rat. ${ }^{6}$ But the

\footnotetext{
${ }^{1}$ Department of Immunemodulation at the Dermatology, University Hospital Erlangen, Erlangen, Germany; ${ }^{2}$ Institute for Medical Microbiology and Hygiene, University Regensburg, Regensburg, Germany and ${ }^{3}$ Nephropathology, Institute for Pathology, University Hospital Erlangen, Erlangen, Germany

Correspondence: Dr A Goldwich, PhD, Department of Immunemodulation University Hospital Erlangen, Hartmannstrasse 14, Erlangen D-91052, Germany.

E-mail: andreas.goldwich@uk-erlangen.de

"These authors contributed equally to this work and should be considered as last author.

Received 25 January 2012; revised 13 August 2012; accepted 23 August 2012
} 
advantage of the genetic manipulation technology to produce a multiplicity of knockouts and transgenics favor the use of murine models. In addition, mice have shorter reproduction periods and are cheaper to maintain. In general, mice do not develop high levels of proteinuria. The maximal rise in proteinuria is only about $3-5$-fold compared with baseline, which is only mild compared with human diseases. Despite this, mouse models have been very helpful to analyze glomerular function, damage and repair mechanisms. The number of murine podocyte injury models, however, is limited so far. Most of these models apply genetically altered mice and induce rapid destruction of podocytes (eg, in case of the NEP $25^{7}$ or the murine Thy $1.1^{8}$ model). Hence, additional easily inducible and robust new mouse proteinuria models operating also in non-transgenic animals are desirable.

Membranous nephropathy (MN) is the leading cause of nephrotic syndrome in adults. Beside the widely used Heymann nephritis model in rats there is only one recently published model of $\mathrm{MN}$ in mice. ${ }^{9,10} \mathrm{Here}, \mathrm{BALB} / \mathrm{c}$ mice were pre-immunized with cationic bovine serum albumin and challenged 2 weeks later i.v. These animals developed features of MN. Unfortunately, mice on a genetic background of C57BL/6, which represent the majority of genetically engineered animals, are resistant to this treatment and thus do no develop proteinuria.

Crescentic glomerulonephritis (GN) is characterized by an extracapillary crescent-shaped proliferation of parietal epithelial cells (PECs) within the urinary space of Bowman's capsule. The corresponding mouse model is the anti-GBM nephrotoxic nephritis. Here, the mice were immunized against sheep or rabbit IgG and the nephritis is induced by the injection of a serum reacting to the whole glomerulus or to the GBM. By day 7, proteinuria starts accompanied by segmental cellular crescents in the glomeruli.

The rat model of puromycin aminonucleoside nephrosis is the experimental prototype of minimal change disease and focal segmental glomerulosclerosis in humans. Later on the rat model of adriamycin (ADR) nephrosis has been established. In both models are the podocytes the side of the initial injury. Although most rat strains are susceptible to both reagents, most mouse strains are not. Exclusively BALB/c mice were found to be susceptible to ADR and develop proteinuria and progressive renal failure. ${ }^{11}$ As mentioned above, many genetically altered mice have a C57BL/6-background and this strain is, as in the case of membraneous nephropathy, also completely resistant to ADR nephrotoxicity.

Beside the models of irreversible glomerular damage and subsequent progressive renal failure, application of lipopolysaccharide (LPS) was described as a model of reversible proteinuria. LPS is the major compound of the outer membrane on Gram-negative bacteria. The binding to the Toll-like receptor 4 (TLR-4) results in an activation of NF- $\kappa \mathrm{B}$ and the initiation of a pro-inflammatory response. Therefore, LPS application was extensively studied as a model for sepsis where proteinuria is an early feature. Reiser et al ${ }^{12,13}$ described a reversible LPS-induced model of proteinuria where intraperitoneal (i.p.) injection of $200 \mu \mathrm{g}$ LPS induced a transient proteinuria within hours returning to baseline after 2 days. The mechanisms show TLR-4 activation on podocytes induces foot process effacement and proteinuria are unknown. The LPS nephrosis model is a sub-septic model affecting podocytes. Caution needs to be exercised when choosing the quality of the LPS and the technique of injection, which can otherwise affect the interpretation of this model.

Thus, in summary further mouse models to analyze the podocyte-specific pattern and mechanism of injury and repair are urgently needed.

In view of the unexpected findings that some mouse strains are sensitive to DTx, independent of the expression of the transgenic primate DTR, we aimed to further investigate how DTx induces death of the animals, which is accompanied by weight loss and proteinuria. Therefore, we analyzed the effect of DTx on kidney function and structure.

\section{MATERIALS AND METHODS}

\section{Animals and Treatments}

C57BL/6 mice, SCID, BALB/c, Rag-2-deficient mice and $\mathrm{C} 3 \mathrm{H} / \mathrm{HeJ}$ mice were obtained from Charles River (Sulzfeld, Germany) or the breeding facility of the University Hospital Erlangen (FPZ). DEREG mice ${ }^{14}$ (gift from Tim Sparwasser, Medizinische Hochschule Hannover, Germany) were used as positive depletion controls. The mice, weighing between 18.6 and $23.3 \mathrm{~g}$, were treated on day 0 and day 1 with $40 \mathrm{ng} / \mathrm{g}$ DTx per bodyweight i.p. (Unnicked, Merck, Darmstadt, Germany). DTx is normally prepared from bacterial lysate. As C. diphtheriae is Gram-positive, no or very low amounts of LPS contamination could be expected. The stock solution of the toxin $(10 \mu \mathrm{g} / \mathrm{ml})$ was contaminated with $0.72 \mathrm{ng} / \mathrm{ml}$ LPS $(72 \mathrm{pg} / \mu \mathrm{g}$ LPS/DTx) as determined with a Limulus Ambocyte Lysate assay (Cambrex, Walkersville, MD, USA). Thus, together with $1.6 \mu \mathrm{g}$ of DTx per mouse $114 \mathrm{pg}$ LPS were administred i.p., which is $\sim 10^{6}$-fold below the dosages used in LPS-proteinuria experiments. ${ }^{13}$ To further exclude undesired LPS effects, we tested heat-treated DTx $\left(95^{\circ} \mathrm{C}\right.$ for $10 \mathrm{~min}$ ) and treated LPS-resistant $\mathrm{C} 3 \mathrm{H} / \mathrm{HeJ}$ mice with DTx.

For comparison we used the established models of the anti-GBM nephritis model and the model of LPS-induced proteinuria. To induce anti-GBM nephritis mice were immunized subcutaneously with $50 \mu$ g rabbit IgG together with $50 \mu \mathrm{l}$ CFA (both obtained from Sigma, Deisenhofen, Germany). After 6 and 7 days rabbit serum (kindly provided by Christian Hugo, University Clinic Dresden, Germany) reacting with the GBM was injected i.v. To induce proteinuria by LPS we injected $10 \mu \mathrm{g} / \mathrm{g}$ mouse weight LPS from Escherichia coli 055:B5 (Sigma L4524) as described. ${ }^{15}$ To detect proteinuria, $5 \mu \mathrm{l}$ of the urine samples were analyzed by Bradford assay together with bovine albumin as standard (both obtained from Bio-Rad, München, Germany). To 
exclude volume variations we pooled the samples on each day and quantified the creatinine concentration. In addition, molecular weight separation of the urinary proteins was done by SDS-polyacrylamide gel electrophoresis followed by silver staining of the gel using standard techniques. Most gels were run under reducing conditions. Gel filtration experiments were additionally performed to analyze proteinuria.

\section{Cell Culture}

HeLa and NIH 3T3 cells were grown for 4 days in Dulbecco's modified Eagle medium (DMEM) plus 10\% v/v fetal calf serum (FCS) with added antibiotics (penicillin, 100 units $/ \mathrm{ml}$; streptomycin $100 \mu \mathrm{g} / \mathrm{ml}$ ). The murine podocyte cell line, ${ }^{16}$ developed from the $\mathrm{H}-2 \mathrm{~K}^{\mathrm{b}}$-tsA58 transgenic mouse strain, ${ }^{17}$ was kindly provided by Karl-Hans Endlich (University of Greifswald, Greifswald, Germany). For permissive conditions, the cells were grown at $33^{\circ} \mathrm{C}$ in RPMI 1640 with FCS $(10 \% \mathrm{v} / \mathrm{v})$, penicillin (100 units $/ \mathrm{ml})$, streptomycin $(100 \mu \mathrm{g} /$ $\mathrm{ml}$ ) and recombinant murine IFN $\gamma(20 \mathrm{ng} / \mathrm{ml}$, PeproTech/ Tebu, Frankfurt, Germany). Differentiating, nonpermissive conditions were achieved by culturing the cells for at least 10 days at $37^{\circ} \mathrm{C}$ in RPMI 1640 supplemented with 5\% FCS with added antibiotics. The preparation and culture of bone marrow cells from C57BL/6 mice to generate dendritic cells (DCs) and macrophages, respectively, has been described previously. ${ }^{18}$ GM-CSF or M-CSF (PeproTech/Tebu) was used at $200 \mathrm{U} / \mathrm{ml}$.

\section{Confocal and Transmission Electron Microscopy}

For subsequent histological analysis the animals were anesthetized and perfusion fixed via the left ventricle. First, icecold PBS was used followed by $4 \%$ paraformaldehyde. The kidneys were taken out and sectioned into 2 -mm thick transverse slices perpendicular to the longitudinal axis. For immunofluorescence renal slices were embedded in paraffin and sectioned. The sections were deparaffinized in xylene and subsequently rehydrated through graded alcohol followed by 5-min incubation with proteinase $\mathrm{K}$ (Roche, Mannheim, Germany, 1:100) to unmask the epitopes. The paraffin sections were then stained with primary antibodies against nephrin (Acris, Herford, Germany), podocin (Sigma) or podocalyxin (R\&D systems, Minneapolis) and Claudin-1 (Abcam, Cambrigde).

After blocking in blotto for $30 \mathrm{~min}$ at room temperature, the primary antibodies $(1: 100)$ were added for $1 \mathrm{~h}$ at $37^{\circ} \mathrm{C}$. Sections were then washed and incubated with Cy2-conjugated secondary antibodies at 1:250 for $30 \mathrm{~min}$ at room temperature. After washing, DAPI was applied for $5 \mathrm{~min}$ and the sections were covered with mounting medium (Dianova, Germany) and evaluated using a confocal microscope (Zeiss LSM 710, together with the software ZEN 2009, Oberkochen, Germany). For transmission electron microscopy, randomly sampled small kidney pieces were fixed in $50 \mathrm{mM}$ sodium cacodylate buffer with $2.5 \%$ glutaraldehyde. The samples were embedded in Epon-Araldite, semithin $(1 \mu \mathrm{m})$ and ultrathin sections $(80 \mathrm{~nm})$ were cut and qualitatively analyzed with an electron microscope (Zeiss EM 109).

\section{Measurement of Cell Vitality by Alamar Blue}

The cells were seeded in 96-well plates 5000/well and treated with different concentrations of DTx as indicated. After $24 \mathrm{~h}$, $20 \mu \mathrm{l}$ alamar blue (AbD Serotec, Düsseldorf, Germany) was added. In this assay, a fluorescent redox indicator was used to monitor the cellular metabolism by detecting changes of the fluorescence monitored at $530-560 \mathrm{~nm}$ excitation wave length and $590 \mathrm{~nm}$ emission wavelength after $24 \mathrm{~h}$. Cells killed by triton X100 $(0.2 \% \mathrm{v} / \mathrm{v})$ were used as negative controls.

\section{Measurement of ${ }^{35} \mathrm{~S}$-Cysteine Incorporation}

The cells were seeded in 24-well plates (100000/well) and treated over night with different concentrations of DTx. Cycloheximide (Sigma), G418 (Invitrogen) and MG132 (Merck) were used as controls. In total, $20 \mu \mathrm{Ci}{ }^{35} \mathrm{~S}$ Protein labeling mix (EasyTag Express, Perkin Elmer, Dreieich) was added for $2 \mathrm{~h}$ at $37^{\circ} \mathrm{C}$. The cells were intensively washed and lysed with $100 \mu$ l lysis buffer. ( $137 \mathrm{mM} \mathrm{NaCl}, 2 \mathrm{mM}$ EDTA, $10 \%$ glycerol, $0.5 \% \mathrm{NP} 40$ and $50 \mathrm{mM}$ Tris $\mathrm{pH} 8.0$ ). An aliquot of the lysat was mixed with scintillation fluid and measured. The untreated controls were set to $100 \%$.

\section{Cell Adhesion to Immobilized Ligands}

Maxisorp (Nunc, Langenselbold, Germany) 96-well flat bottom plates were coated overnight at $4{ }^{\circ} \mathrm{C}$ with fibronectin or collagen type 1 (both obtained from BD Bioscience, San Jose, USA), diluted in $100 \mu \mathrm{l}$ coating buffer $(100 \mathrm{mM} \mathrm{NaCO}$, $\mathrm{pH} 10)$. To block the remaining protein-binding sites on the plate, $100 \mu \mathrm{l} 1.0 \%(\mathrm{w} / \mathrm{v})$ BSA in PBS were added after removing the coating solution and two times washing with PBS $\mathrm{pH}$ 7.4. In each assay, we included wells coated with $1 \%$ BSA alone to determine the background level of cell adhesion. The plates were incubated for $1 \mathrm{~h}$ at $4^{\circ} \mathrm{C}$ and washed twice with PBS.

The podocytes were pretreated with DTx $(100 \mathrm{ng} / \mathrm{ml})$ overnight. Afterwards they were trypsin treated and washed two times with DMEM. The cells were adjusted to $1 \times 10^{6} / \mathrm{ml}$ in attachment buffer (DMEM, $20 \mathrm{mM}$ Hepes, $0.2 \%$ BSA) and were plated (10000 per well) onto the coated wells and incubated at $37^{\circ} \mathrm{C}$ for $2 \mathrm{~h}$. After the cells had been allowed to adhere, the plates were washed gently three times with PBS. The adherent cells were quantified by measuring the activity of acid phosphatase, which is stable, activation independent and not released. To each well $100 \mu$ l lysis buffer $(50 \mathrm{mM}$ sodium acetate, $12 \mathrm{mM}$ p-nitro-phenyl phoshate and $0.2 \%$ $(\mathrm{v} / \mathrm{v})$ Triton $\mathrm{X}-100)$ was added and incubated for $1-2 \mathrm{~h}$ at $37^{\circ} \mathrm{C}$. To determine the cell counts, we used different dilutions of the cells $(10000,5000,2500,1250,625,312$ and 0$)$ as standard. The phosphatase activity was stopped by adding $50 \mu \mathrm{l} 1 \mathrm{~N} \mathrm{NaOH}$. To determine the percentage of cell adhesion the OD was measured with a spectrometric plate reader 
at $405 \mathrm{~nm}$. The statistical analysis was performed using Student's $t$-test or one way ANOVA.

For the real-time attachment assays, we used the RTCA DP Analyzer (Roche) with the same experimental setting as described above. The instrument provides real-time cellular analysis using label-free, noninvasive impedance measuring. The RTCA Analyzer is capable of computer-controlled signal generation, processing and analysis. The measurement rate was $\sim 30 \mathrm{~s}$.

\section{RESULTS}

\section{High Dose of DTx is Lethal for Mice}

DTx is lethal for susceptible animals (including man) in doses of $0.1 \mu \mathrm{g} / \mathrm{kg}$ or less. Owing to the lack of the DTxspecific receptors (heparin-binding EGF-like growth factor) on the cell surface, rodents are much more resistant to DTx. In the transgenic models, the most widely used standard protocol for the DTR-mediated cell depletion is the injection of $40 \mu \mathrm{g} / \mathrm{kg}$ DTx i.p. at once, or on a daily basis for some days. ${ }^{19-21}$ Other investigators use up to $200-300 \mu \mathrm{g} / \mathrm{kg}^{14}$ of the toxin. In Figure 1 we show that C57BL/6 mice tolerate up to $80 \mu \mathrm{g} / \mathrm{kg}$, but at higher doses the animals die even in the absence of the DTR. Of note, in these animals renal histology was normal but urea was markedly increased (data not shown). C57BL/6 mice receiving 225 or $150 \mu \mathrm{g} / \mathrm{kg}$ show a mean survival of 10 or 13 days, respectively. In contrast, the DTR-mediated uptake and cell death is very fast. For example, transgenic mice expressing the DTR in liver cells die within $60 \mathrm{~h}$ after the injection of $0.5 \mu \mathrm{g} / \mathrm{kg}$. ${ }^{4}$ Here, we demonstrate that a high dose of DTx kills the mice by a much slower, so far unknown mechanism, independent of the primate DTR expression. By autopsy we could rule out massive bleeding or major organ pathology. We found, however, that the DTx-treated animals lose weight and develop proteinuria. As high doses of DTx resulted in late lethality and application of lower DTx dosages induced mild weight loss we tested the animals for proteinuria, which could be detected in the majority of animals after DTx application.

\section{DTx Induces Transient and Fully Reversible Proteinuria in Mice}

To further analyze the kinetics and the underlying mechanisms of proteinuria, we performed additional experiments comparing the effects of DTx application with established other models of inducible proteinuria. We found that administration of DTx to DTR-transgenic mice as well as nontransgenic wild-type C57BL/6 mice induced a transient, fully reversible proteinuria. In Figure 2a, we show four exemplified courses of DTx-induced proteinuria of individual mice. The onset of proteinuria varied from day 3 to 7 and its duration differed from 2 to 6 days. Nevertheless, in additional experiments with larger cohorts of mice the majority of DTxtreated animals developed proteinuria (Figures $2 \mathrm{~b}$ and $\mathrm{c}$ ). The disease incidence (defined as proteinuria is once three

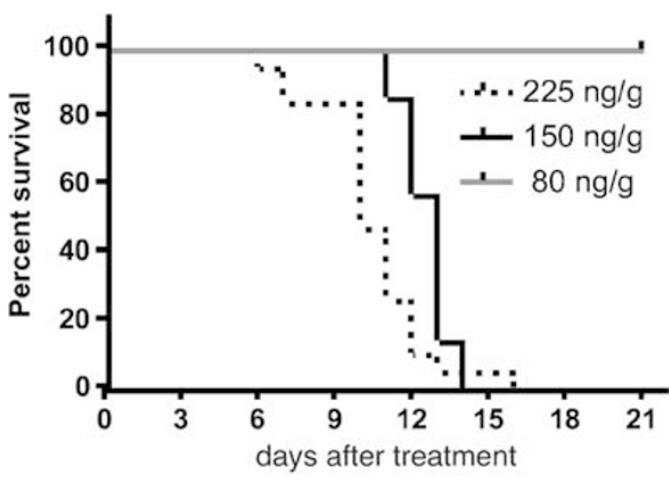

Figure 1 Diphtheria toxin (DTx) dose escalation. The Kaplan-Meier survival curves indicate the toxic potential of DTx. The $225 \mathrm{ng} / \mathrm{g}$ group received three times $75 \mathrm{ng} / \mathrm{g}$ mouse weight intraperitoneal (i.p.; $n=13$ ). The $150 \mathrm{ng} / \mathrm{g}$ group was given three times $50 \mathrm{ng} / \mathrm{g}$ mouse weight i.p. ( $n=8)$ and the $80 \mathrm{ng} / \mathrm{g}$ group received two times $40 \mathrm{ng} / \mathrm{g}$ mouse weight i.p. $(n=7)$. This experiment was repeated several times with similar dose regimens.

times higher as before treatment) among the female animals was slightly, but not significant $(P=0.185)$, higher when compared with males (Figure 2f). Please note, that C57BL/6 mice are very resistant to high levels of proteinuria. As proteinuria (3-5-fold) is mild or modest, we compared the DTx treatment with the so far best known models of non-transgenic proteinuria, ie, the anti-GBM nephritis (Figure 2d) or LPS model (Figure 2e). Of note, the degree of proteinuria in these two models is in the same range, but the incidence of proteinuria after DTx treatment occurred much more reliable. Of further note, LPS treatment resulted in very fast proteinuria within hours (see time scale of Figure 2e) and was accompanied by variable lethality that could only be prevented by i.p. volume substitution.

To exclude that a nonprotein contamination like LPS is responsible for the observed DTx effects, we inactivated the toxin. It is known that the toxin is irreversibly denatured by heat ${ }^{1}$ and as depicted in Figure 3a heat-treated DTx did not induce proteinuria anymore. Vice versa, DTx treatment of LPS-resistant $\mathrm{C} 3 \mathrm{H} / \mathrm{HeJ}$ mice also induced transient proteinuria (Supplementary Figure 1A). As shown in Figure 3b, proteinuria was accompanied by a marked but transient weight loss of the DTx-treated animals. The silver-stained SDS gel in Figure 4 indicates albuminuria as a marker of glomerular and more specifically podocyte damage. In addition, this indicates that proteinuria is not a concentration effect, ie, due to abnormal drinking.

It is well known that tubular reabsorption can compensate glomerular protein loss. The ratio of low-molecular-weight components, like amino acid or peptides $<10000 \mathrm{Da}$ and higher-molecular-weight components, like proteins $>50000 \mathrm{Da}$, indicates either tubular or glomerular damage. In the urine of DTx mice, however, the low-molecular compounds were not altered and only the concentration of proteins $>50 \mathrm{kDa}$ were increased, indicating primary glomerular damage (Figure 4c and Supplementary Figure 2). 

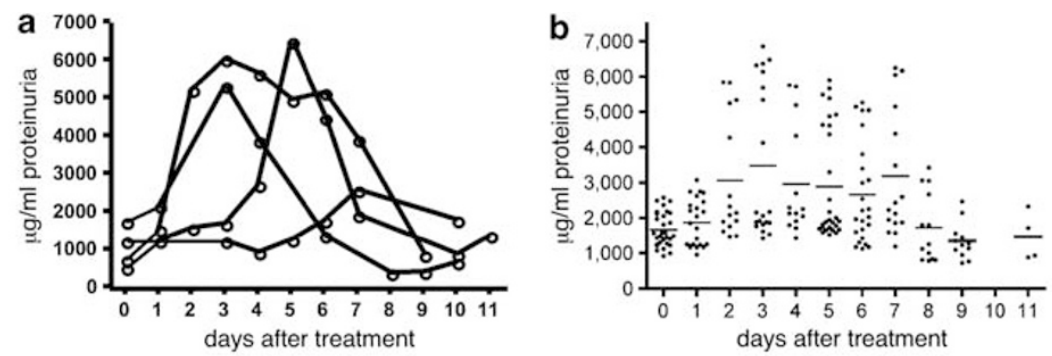

e

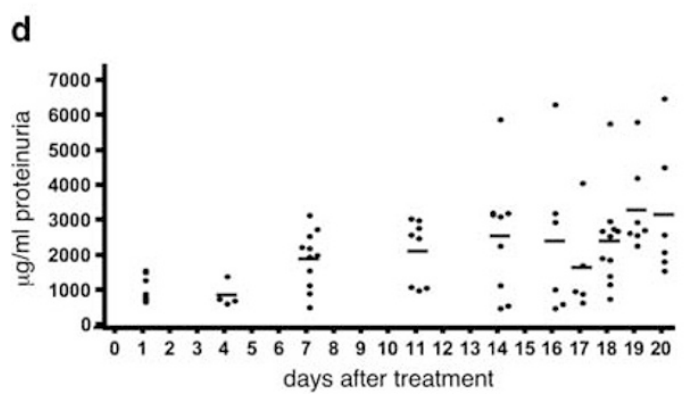

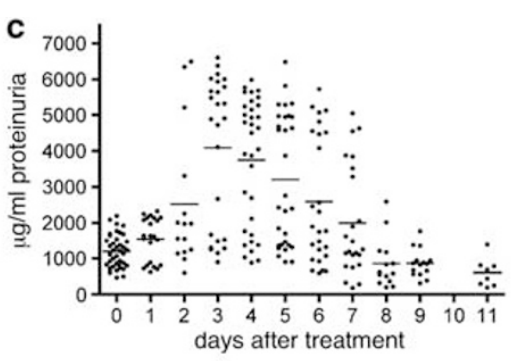

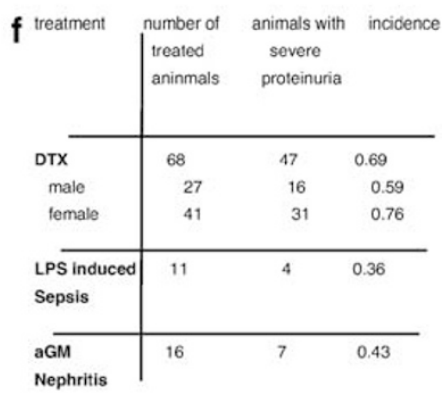

Figure 2 Comparison of different mouse models of proteinuria. Diphtheria toxin (DTx) induces various degrees of reversible proteinuria in mice. C57BL/ 6 mice were treated on day 0 and day 1 with $40 \mathrm{ng} / \mathrm{g}$ bodyweight intraperitoneal (i.p.) The protein content of the urine samples was determined with Bradford assay. Four different clinical courses of proteinuria are demonstrated in (a). All experiments were summarized in (b) (male) and (c) (female). For comparison the clinical courses of anti-GBM nephritis and lipopolysaccharide (LPS)-induced model are shown in (d, e). The calculated incidences of all three proteinuria models are summarized in (f).
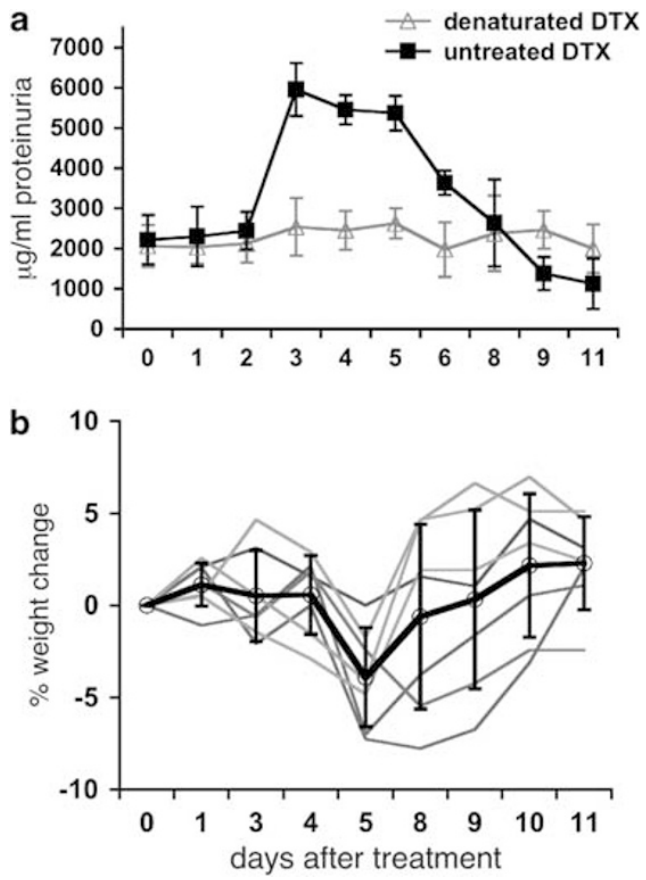

Figure 3 Diphtheria toxin (DTx) induces albuminuria and reversible weight loss. Heat denaturation of DTx disposes the effect (a). This example experiment shows mean and s.d. of $n=7$ untreated DTX and $n=8$ denatured DTx. Mice were weighted before and after DTx treatment and the \% weight change was monitored in (b) (untreated DTX group only).

In the urine of LPS-treated mice excretion of low and highmolecular peptides/proteins was increased indicating mixed glomerular and tubular damage. Interestingly, in the urine of
anti-GBM-treated mice high-molecular-weight proteins were enhanced whereas the low-molecular-weight compounds were reduced.

\section{DTx Induces Podocyte Damage with Foot Processes Effacement and Derangement of Podocytic Proteins}

As albuminuria clearly indicates a glomerular dysfunction, we analyzed if podocyte structure is changed after DTx injection. First, we investigated whether DTx affected glomerular nephrin and podocin expression by confocal microscopy. Nephrin and podocin are important podocytic proteins forming the slit membrane thus being responsible for the maintenance of the glomerular filtration barrier. Figure 5 clearly shows that nephrin as well as podocin expression fades away 48 and $72 \mathrm{~h}$ after DTx injection. At a higher magnification a strong continuous linear podocin stain is observed in untreated, healthy animals indicating the integrity of the glomerular filtration barrier. One or two days after DTx treatment this distinct staining pattern becomes more and more diffuse and podocin-positive blebs appear, which might indicate slit membrane endocytosis. To investigate this in more detail, electron microscopical analyses were performed. Figures $6 \mathrm{a}$ and $\mathrm{b}$ show healthy podocytes at two different magnifications. Please note that the podocytes are tidily attached to the GBM with delicate foot processes. Two days after DTx injection, (Figures $6 c-f)$ mild podocyte damage with foot process effacement (arrows) and enlarged cytoplasm as well as swelling of the glomerular endothelium (white asterisk) is seen. Of note, no alterations of the GBM are present and there was no correlation between endothelial swelling and podocyte damage, 

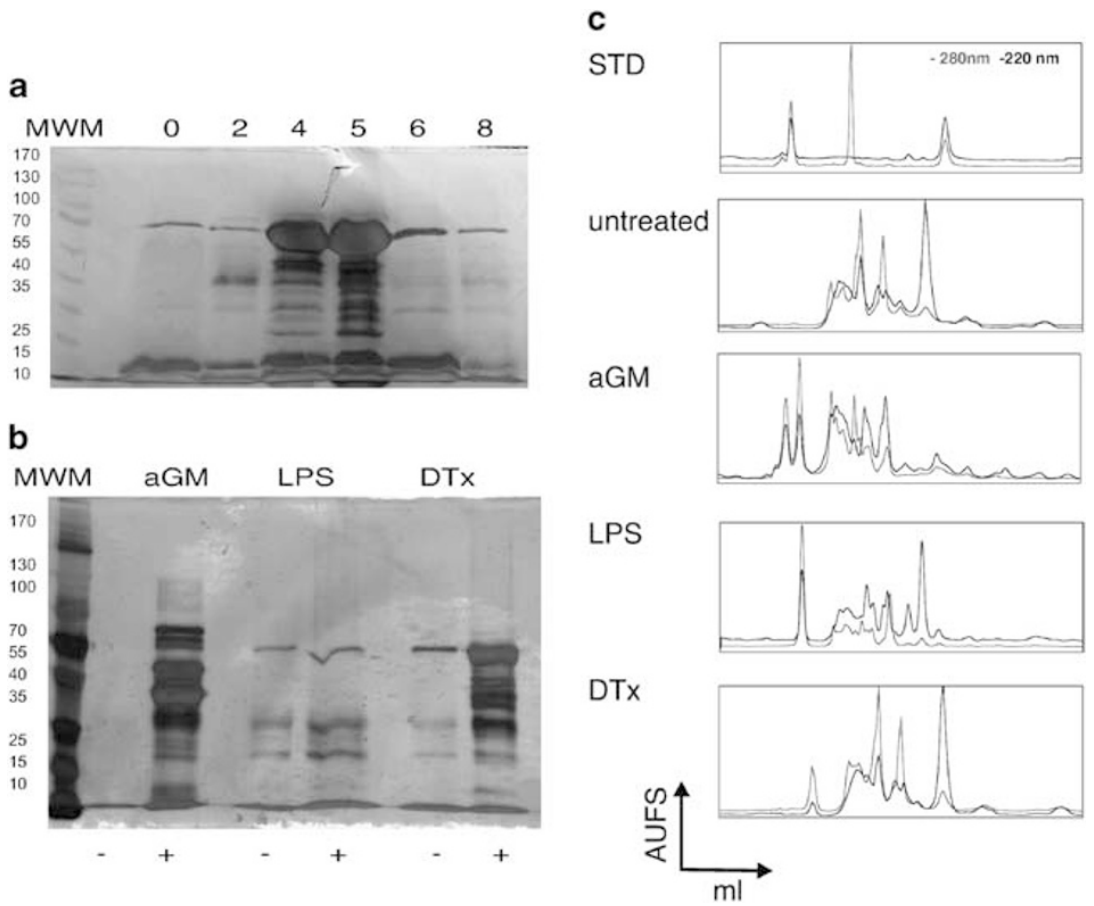

Figure 4 Diphtheria toxin (DTx) induces albuminuria. C57BL/6 mice were treated on day 0 and day 1 with $40 \mathrm{ng} / \mathrm{g}$ bodyweight DTx intraperitoneal (i.p.). (a) Two microliter urine was separated on $8 \%$ sodium dodecyl sulfate (SDS) gels. An exemplified silver-stained SDS gel indicates marked albuminuria. (b) For comparison urine samples of the anti-glomerular basement membrane (GBM) nephritis and the lipopolysaccharide (LPS)-induced model are also shown. The two lanes show the proteins in the urine before and at the peak of the disease. (c) Analysis of proteinuria by gel filtration. The diluted urine samples at the peak of proteinuria were separated on a superdex 75 column. Albumine (68000 Da), the peptide OVA 323-339 $(1760 \mathrm{Da})$ and the amino-acid tryptophan $(204 \mathrm{Da})$ were use as standards.

suggesting that DTx may directly injured endothelial cells. There is no doubt that these structural alterations together with the accompanying changes in electrical charge may induce proteinuria in the DTx-treated animals. After resolution of proteinuria, no difference in glomerular ultrastructure could be detected between the DTx-treated or -untreated animals (Supplementary Figure 3).

\section{DTx Does not Influence the Viability of Murine Podocytes}

As DTx induces a morphological change of the podocytes, we analyzed in complementary in vitro studies if the podocytes were killed or harmed by the toxin. Conditionally immortalized podocytes were incubated with various amounts of DTx, LPS or a sheep serum reacting against murine podocytes. $^{22}$ Figure $7 \mathrm{a}$ and Supplementary Figure $4 \mathrm{~A}$ show that despite high concentration of DTx the podocytes do not lose viability. When this experiment was repeated with isolated podocytes from murine kidneys we found the same result. Human HeLa cells served as positive and murine fibroblast cell line NIH 3T3 as negative controls. One interpretation of this finding is that the molecule, which functions as a receptor for DTx in vivo, may not be expressed in cultured podocytes. However, in view of the results of the below mentioned attachement assay this seems unlikely. Compared with human HeLa cells the protein biosynthesis of murine podocytes was not inhibited (Figure 7b) by DTx. In both cell lines the ${ }^{35} \mathrm{~S}$-cysteine incorporation was reduced to $20 \%$ in the presence of cycloheximide and G418. These residual counts represent the amino-acid uptake into the cytosol. MG132 normally used as proteasome inhibitor also decreased the protein biosynthesis, if it was present for $>30 \mathrm{~min}^{23}$ (Figure 7c).

As phagocytic cells such as macrophages or immature DCs might also take up and internalized some of the toxin this side may induce proteinuria. When we repeated the above experiments in murine DCs and macrophage from C57BL/6 mice, we found these cells to be also resistant to DTx (Supplementary Figure 4B). Next, we analyzed if the number of podocytes per glomerular area changes by using WT-1, a highly specific transcription factor as podocytic marker. We could show that the number of WT-1-positive cells, ie, podocytes, is not altered during DTx treatment (Supplementary Figure 5) ruling out podocyte apoptosis or proliferation. Additional stainings with antibodies to activated caspase- 3 as an apoptotic marker and Ki-67 as a proliferation marker are negative (data not shown). These data indicate that the podocytes were not killed by the toxin and that proteinuria is most likely due to a cellular change or altered podocyte function.

DTx Alters Attachment of Podocytes to the GBM In Vitro To affirm the morphological observations, attachment assays were performed using 96-well plates coated overnight with 
collagen 1 or fibronectin. In parallel, podocytes were treated with $100 \mathrm{ng} / \mathrm{ml}$ DTx or left untreated. The percentage of untreated cells attaching to fibronectin or collagen-1 as substrate was significantly higher $(P<0.001$ for concentration $>1 \mu \mathrm{g} / \mathrm{ml}$ substrate) compared with the DTx-treated podocytes (Figures $8 \mathrm{a}$ and $\mathrm{b}$ ). Beside the classical attachment assay real-time attachment assays using the RTCA DP
(Roche) were performed. The impedance chance in the chamber correlates with the number of cells attaching and spreading to the coated chamber bottom and this was indicated by the cell index (Figures $8 \mathrm{c}$ and d). Furthermore, it was possible to calculate the EC50, ie, the concentration of the adhesion substrate that allows $50 \%$ of the maximal attained attachment. In the case of fibronectin it was
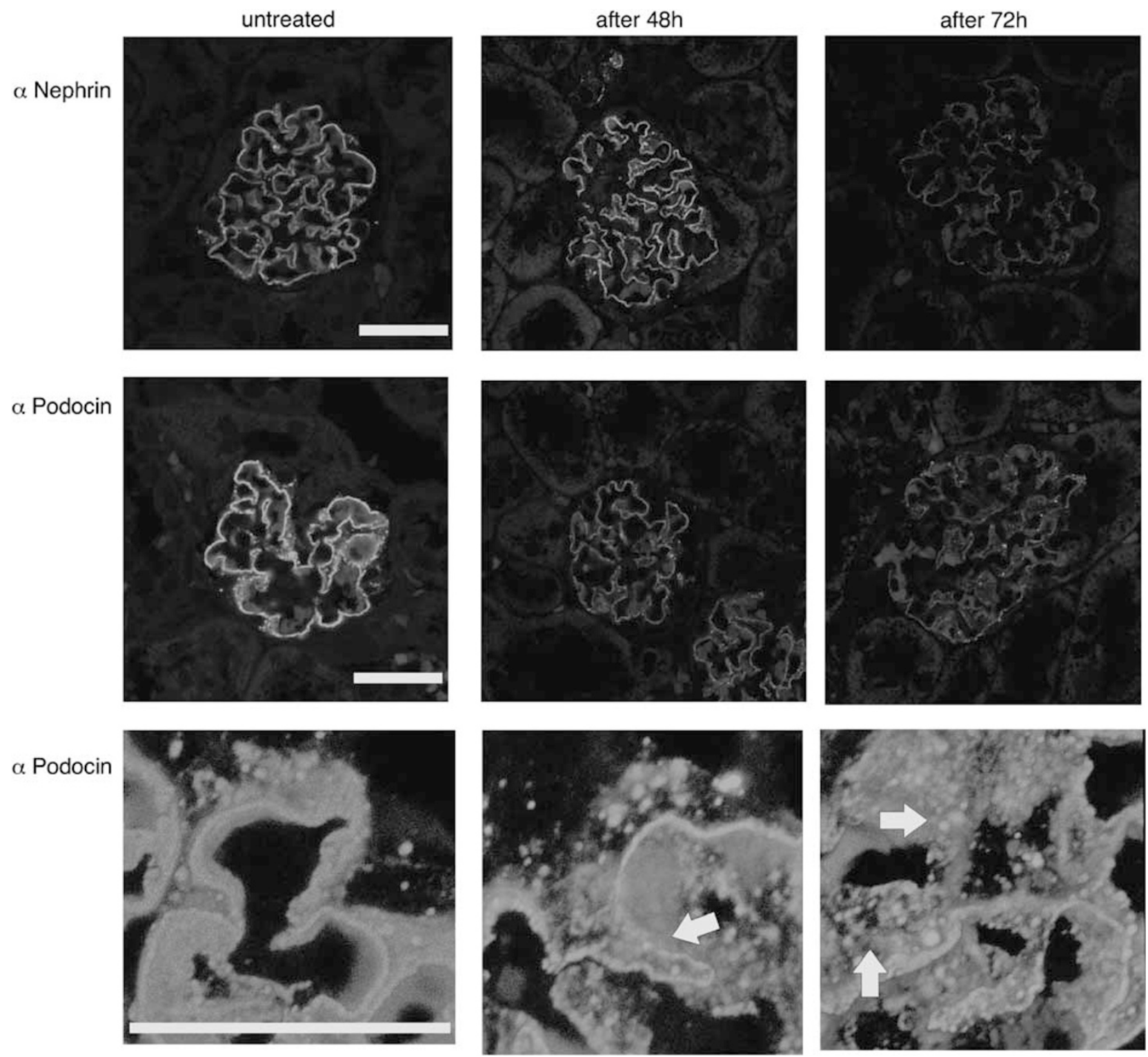

Figure 5 Confocal microscopy of nephrin and podocin immunofluorescence. Compared with untreated animals, glomerular nephrin (first row) and podocin staining is markedly lower 48 and $72 \mathrm{~h}$ after diphtheria toxin injection. As seen at a higher magnification (third row) more diffuse and weaker podocin staining is observed together with some blebbing (yellow arrow). The yellow magnification bar indicates $20 \mu \mathrm{m}$.

Figure 6 Glomerular ultrastructure of diphtheria toxin (DTx)-treated (c-f) and control (a, b) mice. On electron microscopy after DTx injection (c-f) mild podocyte damage with effacement of the foot processes (arrows) and enlarged cytoplasm as well as swelling of the glomerular endothelium (white asterisk) is seen compared with control animals where foot processes and endothelial cells are well maintained (a, $\mathbf{b})$. Of note, no alterations of the GBM are seen. Magnifications: $\mathbf{a}, \mathbf{c}: \times 7750 ; \mathbf{b}, \mathbf{d}: \times 27800$; and $\mathbf{e}, \mathbf{f}: \times 16700$. 

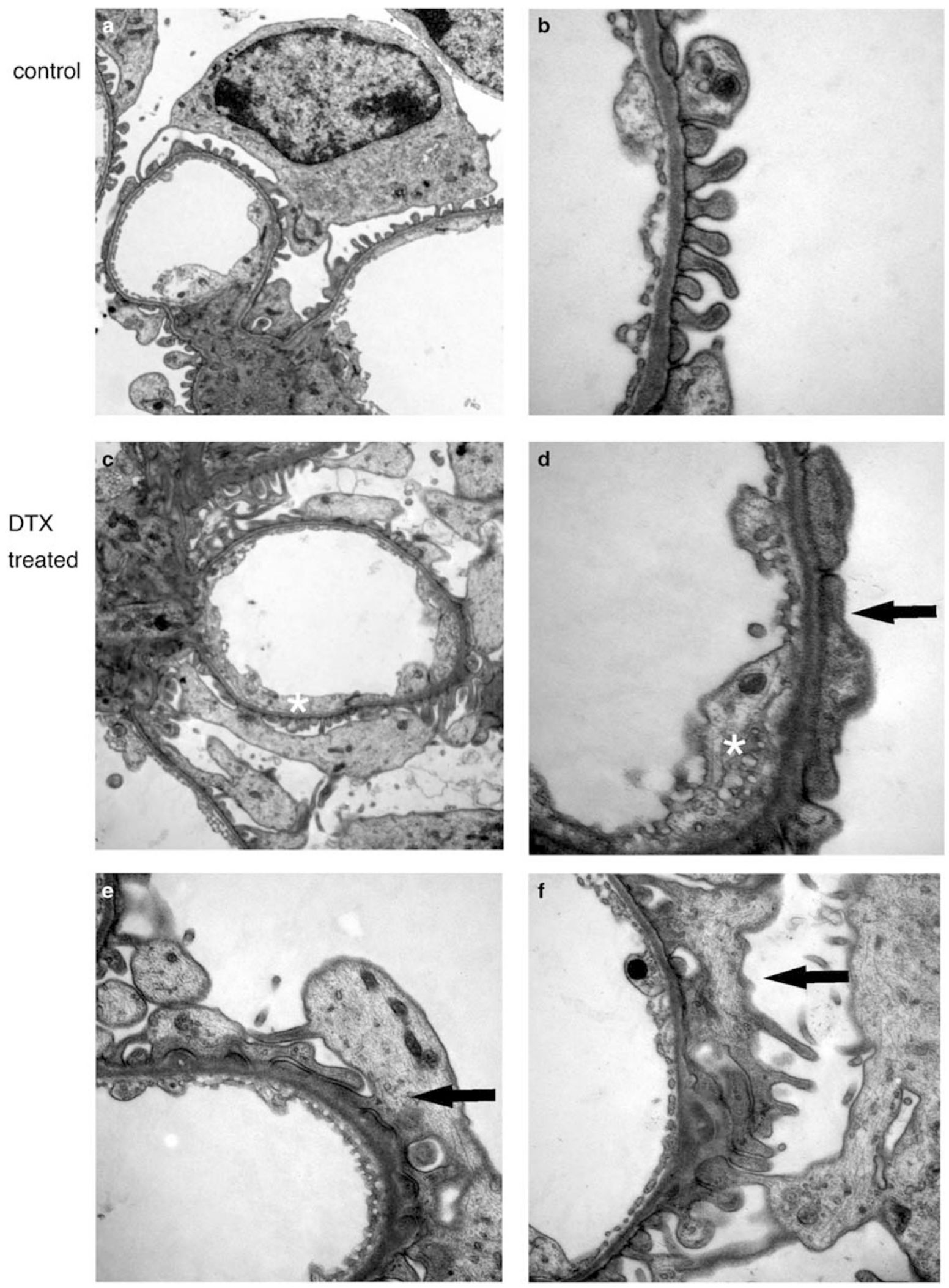

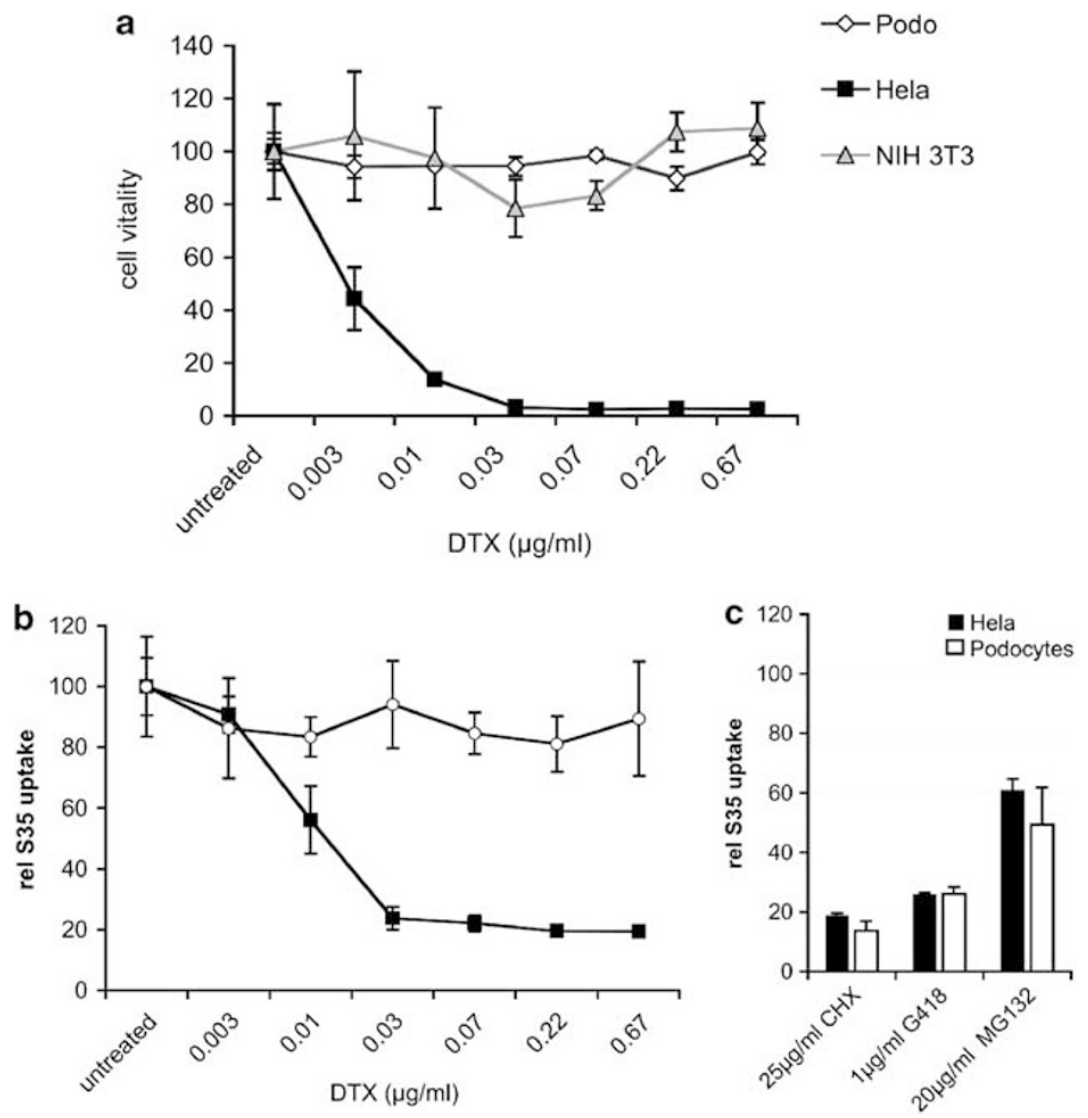

Figure 7 Cell viability after diphtheria toxin (DTx) treatment in vitro. Conditional immortalized murine podocytes, a murine fibroblast cell line and a human control cell (cervix carcinoma) were treated with the indicated concentrations of DTx. (a) After $24 \mathrm{~h}$, alamar blue was added and the cell vitality was determined by fluorometry after additional $24 \mathrm{~h}$. Untreated cells served as $100 \%$ control and Triton lysed cells as background (0\% living cells). (b, c) $20 \mu \mathrm{Ci}$ of $35 \mathrm{~S}$-protein labeling mix were added for $2 \mathrm{~h}$ at $37^{\circ} \mathrm{C}$. The cells were intensively washed and lysed with $100 \mu \mathrm{ll}$ lysis buffer. Cyclohexemid, G418 and MG132 were used as controls. Ten microliter of the lysat was mixed with scintillation fluid and measured. The untreated controls were set to $100 \%$. These experiments were repeated at least three times.

$0.047 \mu \mathrm{g} / \mathrm{ml}$ for untreated cells and $0.160 \mu \mathrm{g} / \mathrm{ml}$ for DTxtreated podocytes, indicating that DTx-treated podocytes need 3.4 times more substrate to adhere. Usually, adhesion force negatively correlates with cell mobility. Velocity measurements shown in Supplementary Figure 6 indicated that cell mobility is slightly, but not significantly, enhanced in the presence of DTx or LPS.

In summary, these experiments underline our morphological findings in kidneys of mice, ie, that DTx interferes with podocyte structure and attachment and leads to an inchoate filtration barrier and albuminuria. Owing to the fact that podocytes survive DTx administration recovery seems possibly and explains the complete resolution of proteinuria after 5 or 7 days.

\section{DISCUSSION}

We describe a new reversible model of mild and reversible experimental podocyte injury in mice by injection of DTx. Here, proteinuria is due to podocyte dysfunction characterized by foot process effacement and derangement of podocytic proteins such as nephrin and podocin. The DTx model is easy to handle and works with a high reliability. Owing to the comprehensive vaccination program in the western world DTx is not at all dangerous for the investigator. Many genetically altered mice are on a C57BL/6 background and this strain is known to be resistant to most renal injury models. By using DTx it is now possible to induce transient proteinuria in this strain and its multiple genetically altered substrains. However, the maximal rise in proteinuria is only about 3-5-fold compared with baseline, which is only mild compared with human diseases with about 1000 -fold changes or rat models with up to 50 -fold changes. It is noteworthy that mice in general are more resistant to higher levels of proteinuria. It is of note, however, that the range of proteinuria in the DTx model is within the same range as in other murine proteinuria models. We therefore compared this new model with the most widely used podocyte damage models, ie, the anti-GBM nephritis and the LPS-induced proteinuria model, which are accompanied by an only moderate incidence of proteinuria. Moreover, these two models harbor additional drawbacks, ie, LPS-induced proteinuria develops very fast and a significant proportion of the animals would 


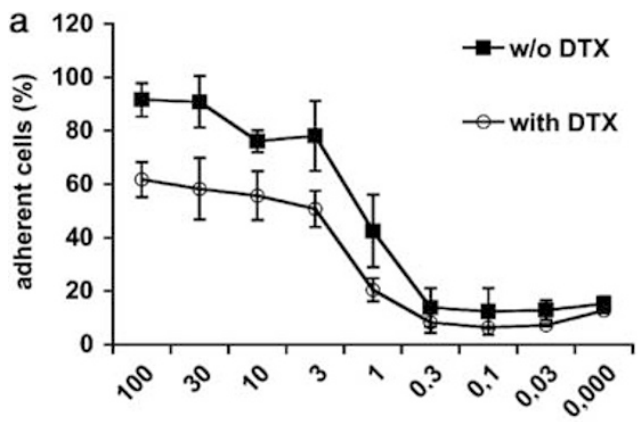

Fibronectin $(\mu \mathrm{g} / \mathrm{ml})$

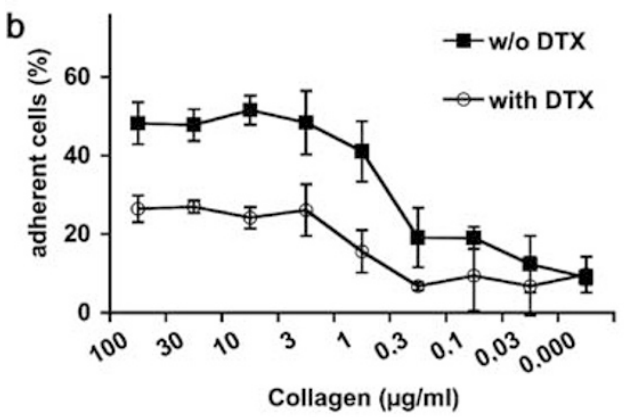

C

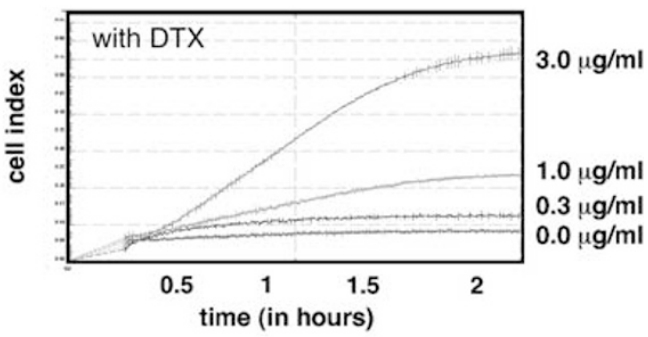

d

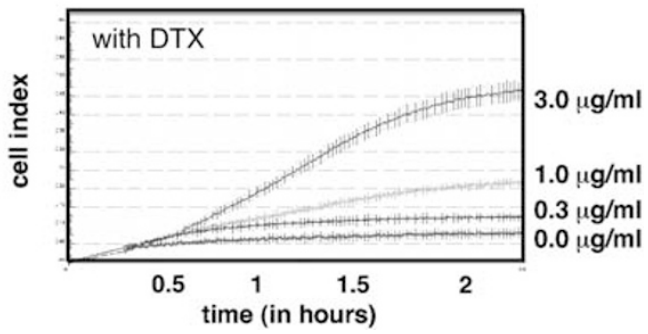

Figure 8 Attachment assays. The podocytes were treated for $24 \mathrm{~h}$ with $100 \mathrm{ng} / \mathrm{ml}$ diphtheria toxin (DTx) and used either in a conventional adhesion assay on MaxiSorp flat bottom 96-well plates coated with serial dilution ranging from $33 \mathrm{ng} / \mathrm{ml}$ up to $100 \mu \mathrm{g} / \mathrm{ml}$ of the respective protein, or in the real-time adhesion assay using an RTCA DP Analyzer. For the conventional cell adhesion assays $10^{4}$ cells per well were plated on coated wells and incubated at $37^{\circ} \mathrm{C}$ for $2 \mathrm{~h}$. The number of adherent cells was analyzed by measuring the activity of acid phosphatase. For calibration, in parallel experiments activity of acid phosphatase was measured with defined numbers of cells $(10000,5000,2500,1250,625$ and 312) that were added to additional wells on the same ELISA plate. Means \pm s.d. of eight wells were calculated and statistical analysis was performed using an unpaired Student's $t$-test $(P<0.05)$. For each experiment, eight wells (corresponding to one lane) were used for each concentration and protein. Each experiment was repeated at least three times. The real-time attachment was done with the RTCA DP Analyzer according to the protocols of the manufacturer. die without volume reconstitution. Analysis of glomerular transcriptome changes induced by LPS via TLR- 4 showed that many immunological gene categories like immune response genes, defense genes, cytokine genes and many others are upregulated in the LPS-treated animals. ${ }^{24}$ Therefore, it is apparently very difficult in this model to separate primary podocyte dysfunction from immunological sepsis side effects. Similar side effects are seen in the anti-GBM nephrits. Glomerular damage is induced by immunocomplex deposition along the GBM, followed by complement activation, inflammation and crescent formation. Therefore, many immunological factors may influence the progressive course of the disease. Finally, this model is lethal for the animal and thus mechanisms of functional restoration cannot be studied.

DTx application is an interesting new tool to transiently modify podocyte functions without ablating these cells and without any signs of renal inflammation on histology. Of note, DTx injection also induced proteinuria in immunological compromised mice strains without $\mathrm{T}$ and $\mathrm{B}$ cells, ie, SCID or rag $1-/-$ mice (Supplementary Figures 1B and C). Thus, the DTx model provides the great opportunity and advantage to analyze responses to the local damage without any immunological interaction. Although this animal model has some features of a mild podocytopathy such as in minimal change nephropathy, care should be taken to adopt experimental mouse data directly into human glomerular diseases. Nevertheless, our model offers a new opportunity to analyze the renal response to injury and the compensating regenerative processes.

It has been demonstrated recently ${ }^{25,26}$ that PECs are the progentitor cells that are able to regenerate podocytes after injury. In our in vivo model, we did not detect activated PECs by claudin-1/podocalyxin double staining. Furthermore, the number of podocytes was not changed during development and resolution of proteinuria. Thus, we assume a resolution of podocyte damage instead of a regenerative process by progentitor cells. It is not completely clear, however, how the toxin interacts with the podocytes. The molecular mechanisms underlying foot processes effacement of podocytes and detachment from the GBM substrated in vivo and in vitro without affecting viability of the cells are ill defined and are currently under more detailed investigation in our laboratory. We show that the inactivation of eEF2 and the subsequent inhibition of protein synthesis are below the threshold. Not mutually exclusive, alternative DTRindependent mechanisms of DTx uptake into subcellular compartments could result in cytoskeletal or other changes that could disturb the podocyte-matrix interaction, thereby inducing proteinuria. We would like to point out, however, that the cell attachment is only reduced but not completely abolished by DTx. This indicates that additional mechanisms could be involved. Cell vitality measurements with podocytes or other highly phagocytic murine cells such as DCs or macrophages show that these cells are also resistant against 
DTx. This is of particular interest as the uptake of a single DTx molecule could be sufficient to kill a cell. ${ }^{27}$ Therefore, we assume an additional extra cellular target of DTx on podocytes and also on other cells. There are reports from resistant mouse and rat cells, that these cells bind the toxin. ${ }^{28-30}$ Irrespective of the molecular mechanism involved DTx-induced transient podocyte malfunction and proteinuria might be a supplemental new and convenient experimental model, which can also be applied to the great variety of genetically engineered C57BL/6 mice. Of note, $\mathrm{BALB} / \mathrm{c}$ mice get sick as well but the magnitude of proteinuria is lower than in C57BL/6 mice (Supplementary Figure 1D).

The knowledge that DTx induces podocyte injury should be considered in the future when interpreting results with DTx depleter mice for hypertension or renal disease models. For example, it has been shown that administration of DTx to transgenic mice that express the DTR under the control of the CD11c promotor resulted in the depletion of CD11c + DCs. The depletion of DCs resulted in a complete inability to cross-prime $\mathrm{CD} 8+\mathrm{T}$-cell responses in various models ${ }^{21}$ including the activation of autoreactive CTLs in the renal lymph nodes by renal DCs. This activation normally causes GN and DC depletion resolved or prevented the disease. ${ }^{31,32}$ First limitations for the conditional DC depletions in CD11c-DTR transgenic mice have been reported by Probst et $a l^{20}$ They investigated the depletion of DCs in CD11c-DTR mice and unexpectedly found a complete and long-lasting depletion of marginal zone and metallophilic macrophages in the spleen and lymph nodes due to ectopic CD11c expression beside the transient depletion of CD11c + DCs. Hence, the authors questioned the use of CD11c DTR mice for the analysis of DCs function, because it could not strictly be separated from effects on certain macrophage subsets.

On the other side, depletion of macrophages in CD11bDTR mice halts the progression of anti-GBM nephritis. ${ }^{33}$ The depletion of macrophages by DTx at the time point of an already beginning progressive disease reduced the number of glomerular crescents. Interestingly, a temporary enhanced proteinuria was seen, but this did not further increase compared with control animals. These findings nicely fit to our data where DTx induces a primary reversible proteinuria due to its effects on podocytes, whereas the depletion of macrophages halts the progression of kidney tissue damage. In a recent publication Ooi $e a^{34}$ showed that depletion of Tregs in foxp3 DTR mice increased renal injury in the antiGBM nephrits model. There is no doubt that Tregs are very important to suppress inflammation, whereas it could not be excluded that podocyte injury by DTx could additionally also amplify the disease.

In many DTR-transgenic mouse models specific depletion of DTR-expressing cells was seen 1-2 days after DTx treatment. The targeted cell population recovered after 6 days for DCs ${ }^{21}$ after 3 days in the case of macrophages ${ }^{33}$ or after
10 days when Tregs were depleted. ${ }^{14}$ In our view, however, conclusions from experiments using DTR-transgenic mice have to be interpreted with caution for those models that involve podocyte functions, which might be especially relevant for mouse models to study hypertension or kidney malfunctions.

Supplementary Information accompanies the paper on the Laboratory Investigation website (http://www.laboratoryinvestigation.org)

\section{ACKNOWLEDGEMENTS}

We thank Martha Ölke, Nadine Pangratz, Miriam Reutelshöfer, Susanne Rößner and Christina Saemann for excellent technical support, and Leonie Littmann for the support as veterinarian. This work was supported by the Deutsche Forschungsgemeinschaft (SFB 796 B2, SFB 423 Z2, SFB 643 and Ge 671/12-2) and the Interdisciplinary Center for Clinical Research (IZFK, projects A45 and F1) at the University Hospital Erlangen-Nuremberg.

\section{DISCLOSURE/CONFLICT OF INTEREST}

The authors declare no conflict of interest.

1. Pappenheimer Jr AM. Diphtheria toxin. Annu Rev Biochem 1977;46: 69-94.

2. Mekada $\mathrm{E}$, Senoh $\mathrm{H}$, Iwamoto $\mathrm{R}$, et al. Purification of diphtheria toxin receptor from Vero cells. J Biol Chem 1991;266:20457-20462.

3. Naglich JG, Metherall JE, Russell DW, et al. Expression cloning of a diphtheria toxin receptor: identity with a heparin-binding EGF-like growth factor precursor. Cell 1992;69:1051-1061.

4. Saito M, Iwawaki T, Taya C, et al. Diphtheria toxin receptor-mediated conditional and targeted cell ablation in transgenic mice. Nat Biotechnol 2001;19:746-750.

5. Obeidova H, Merta M, Reiterova J, et al. Genetic basis of nephrotic syndrome—review. Prague Med Rep 2006;107:5-16.

6. Pippin JW, Brinkkoetter PT, Cormack-Aboud FC, et al. Inducible rodent models of acquired podocyte diseases. Am J Physiol Renal Physiol 2009;296:F213-F229.

7. Matsusaka T, Xin J, Niwa S, et al. Genetic engineering of glomerular sclerosis in the mouse via control of onset and severity of podocytespecific injury. J Am Soc Nephrol 2005;16:1013-1023.

8. Smeets B, Dijkman HB, te Loeke NA, et al. Podocyte changes upon induction of albuminuria in Thy-1.1 transgenic mice. Nephrol Dial Transplant 2003;18:2524-2533.

9. Chen JS, Chen A, Chang LC, et al. Mouse model of membranous nephropathy induced by cationic bovine serum albumin: antigen dose-response relations and strain differences. Nephrol Dial Transplant 2004;19:2721-2728.

10. Wu CC, Chen JS, Chen SJ, et al. Kinetics of adaptive immunity to cationic bovine serum albumin-induced membranous nephropathy. Kidney Int 2007;72:831-840.

11. Chen A, Wei $\mathrm{CH}$, Sheu LF, et al. Induction of proteinuria by adriamycin or bovine serum albumin in the mouse. Nephron 1995;69: 293-300.

12. Wei C, Moller CC, Altintas MM, et al. Modification of kidney barrier function by the urokinase receptor. Nat Med 2008;14:55-63.

13. Faul C, Donnelly M, Merscher-Gomez S, et al. The actin cytoskeleton of kidney podocytes is a direct target of the antiproteinuric effect of cyclosporine A. Nat Med 2008;14:931-938.

14. Lahl K, Loddenkemper C, Drouin C, et al. Selective depletion of Foxp3 + regulatory T cells induces a scurfy-like disease. J Exp Med 2007;204:57-63.

15. Reiser J, von Gersdorff G, Loos M, et al. Induction of B7-1 in podocytes is associated with nephrotic syndrome. J Clin Invest 2004;113: 1390-1397.

16. Schiwek D, Endlich N, Holzman L, et al. Stable expression of nephrin and localization to cell-cell contacts in novel murine podocyte cell lines. Kidney Int 2004;66:91-101. 
17. Jat PS, Noble MD, Ataliotis $P$, et al. Direct derivation of conditionally immortal cell lines from an $\mathrm{H}-2 \mathrm{~Kb}$-tsA58 transgenic mouse. Proc Natl Acad Sci USA 1991;88:5096-5100.

18. Lutz MB, Kukutsch N, Ogilvie AL, et al. An advanced culture method for generating large quantities of highly pure dendritic cells from mouse bone marrow. J Immunol Methods 1999;223:77-92.

19. Duffield JS, Tipping PG, Kipari $T$, et al. Conditional ablation of macrophages halts progression of crescentic glomerulonephritis. Am J Pathol 2005; 167:1207-1219.

20. Probst HC, Tschannen K, Odermatt B, et al. Histological analysis of CD11c-DTR/GFP mice after in vivo depletion of dendritic cells. Clin Exp Immunol 2005;141:398-404.

21. Jung S, Unutmaz D, Wong $\mathrm{P}$. et al. In vivo depletion of CD11C + dendritic cells abrogates priming of CD8 + T cells by exogenous cellassociated antigens. Immunity 2002;17:211-220.

22. Meyer-Schwesinger $C$, Dehde $S$, Klug $P$, et al. Nephrotic syndrome and subepithelial deposits in a mouse model of immune-mediated antipodocyte glomerulonephritis. J Immunol 2011;187:3218-3229.

23. Schubert U, Anton LC, Gibbs J, et al. Rapid degradation of a large fraction of newly synthesized proteins by proteasomes. Nature 2000 ; 404:770-774.

24. Sun $\mathrm{Y}, \mathrm{He} \mathrm{L}$, Takemoto $\mathrm{M}$, et al. Glomerular transcriptome changes associated with lipopolysaccharide-induced proteinuria. Am J Nephro 2009;29:558-570.

25. Smeets B, Uhlig S, Fuss A, et al. Tracing the origin of glomerular extracapillary lesions from parietal epithelial cells. J Am Soc Nephrol 2009;20:2604-2615.
26. Appel D, Kershaw DB, Smeets B et al. Recruitment of podocytes from glomerular parietal epithelial cells. J Am Soc Nephrol 2009;20:333-343.

27. Yamaizumi M, Mekada E, Uchida T, et al. One molecule of diphtheria toxin fragment A introduced into a cell can kill the cell. Cell 1978; 15:245-250.

28. Didsbury JR, Moehring JM, Moehring TJ. Binding and uptake of diphtheria toxin by toxin-resistant Chinese hamster ovary and mouse cells. Mol Cell Biol 1983;3:1283-1294.

29. Chang T, Neville Jr DM. Demonstration of diphtheria toxin receptors on surface membranes from both toxin-sensitive and toxin-resistant species. J Biol Chem 1978;253:6866-6871.

30. Keen JH, Maxfield FR, Hardegree MC, et al. Receptor-mediated endocytosis of diphtheria toxin by cells in culture. Proc Natl Acad Sci USA 1982;79:2912-2916.

31. Heymann F, Meyer-Schwesinger C, Hamilton-Williams EE, et al. Kidney dendritic cell activation is required for progression of renal disease in a mouse model of glomerular injury. J Clin Invest 2009;119:1286-1297.

32. Dong X, Swaminathan S, Bachman LA, et al. Antigen presentation by dendritic cells in renal lymph nodes is linked to systemic and local injury to the kidney. Kidney Int 2005;68:1096-1108.

33. Goren I, Allmann N, Yogev N, et al. A transgenic mouse model of inducible macrophage depletion: effects of diphtheria toxin-driven lysozyme M-specific cell lineage ablation on wound inflammatory, angiogenic, and contractive processes. Am J Pathol 2009;175:132-147.

34. Ooi JD, Snelgrove SL, Engel DR, et al. Endogenous foxp3(+) T-regulatory cells suppress anti-glomerular basement membrane nephritis. Kidney Int 2011;79:977-986. 